\title{
Salmonella meningitis following treatment of enteritis with neomycin
}

\author{
C. HARDY \\ M.B., B.S., B.Sc., M.R.C.P. \\ J. A. LOWES \\ M.B., B.S., M.R.C.Path.
}

A. BANSAL

B.M.

C. F. GEORGE

B.Sc., M.D., F.R.C.P.

Southampton General Hospital, Tremona Road, Southampton S09 4XY

\begin{abstract}
Summary
A case of salmonella meningitis with accompanying septicaemia and pericarditis is reported in an adult following an episode of enteritis.
\end{abstract}

KEY WORDS: food poisoning, pericarditis, septicaemia, ampicillin, chloramphenicol.

\section{Introduction}

The first documented case of salmonella meningitis was reported by Ghon in 1907. It is now recognized that meningitis is a rare complication of infection with salmonella organisms and occurs almost exclusively in infants (Beene, Hansen and Fulton, 1951; Saphra and Winter, 1957). We report a case of salmonella meningitis in an adult. Not only was his presentation unusual in that the illness occurred abruptly during the convalescent phase of an attack of salmonella enteritis, but also he showed evidence of concurrent pericarditis, a combination which has not hitherto been described.

\section{Case report}

A 17-year-old school boy was admitted to hospital with a 12-hr history of increasing headache, drowsiness and persistent vomiting. One month previously he had been on holiday in Ibiza where he had developed diarrhoea with the passage of mucus but not blood per rectum: there was no history of abdominal pain. However, the diarrhoea persisted for 2 weeks and because of this he consulted his general practitioner on returning to England. A species of salmonella was cultured from his stool and he was treated for this with a 6-day course of oral neomycin $500 \mathrm{mg}$ qds. Subsequently, two negative stool cultures were obtained. For 10 days he remained well until the day of his admission. Although no other member of the family had symptoms, the same enteritic organism was recovered in the stools of his father.

On admission he had a temperature of $39^{\circ} \mathrm{C}$ and tachycardia of 120 beats $/ \mathrm{min}$. He was drows9 irritable and had marked neck stiffness. There were no papilloedema or focal neurological signs detected Examination also revealed a loud pericardial friction rub and that the spleen was palpable $3 \mathrm{~cm}$ below the costal margin with a firm consistency.

Lumbar puncture demonstrated turbid cerebrospinal fluid (CSF) at a pressure of $270 \mathrm{mmH}_{2} \mathrm{O}$. Sce Gram-negative bacilli were noted and subsequentl on culture a salmonella species with an identicas agglutination pattern to that cultured previouslo from his stools was grown. CSF and blood glucose measurements were 0.15 and $7.6 \mathrm{mmol} / 1$ respectivel $\overrightarrow{\mathrm{H}}$ and the CSF protein was elevated at $3,900 \mathrm{mg} / \mathrm{l}$ : the CSF white cell count was $2.9 \times 10^{9} / 1$, all polymorph The peripheral blood count showed a polymorp leucocytosis of $17.8 \times 10^{6} / 1$ but blood and urine cultures were repeatedly sterile. The electrocardio 3 gram (ECG) showed a partial right bundle branci block pattern with $\mathrm{T}$ wave inversion in leads $\mathrm{V} 1$ and V2.

A presumptive diagnosis of salmonella meningitis, secondary to septicaemia from salmonella enteritio was made, and therapy commenced with intravenous chloramphenicol $4.8 \mathrm{~g}$ daily. However, since the patient failed to improve after $12 \mathrm{hr}$ on this treatment (plus symptomatic aspirin therapy) and the organisp? had been contracted in Ibiza (where chloramphenicol is widely used) ampicillin was added $12 \mathrm{hr}$ later. The initial dose was $4 \mathrm{~g}$ but this was later increased to 12 $\mathrm{g} /$ day. There was an immediate improvement follows ing the addition of ampicillin but the patient's fevef persisted and the pericardial friction rub was audible for 7 days. The chloramphenicol was stopped after 7 days and the ampicillin after 2 weeks. Just before its 
withdrawal, a second lumbar puncture was performed at which the CSF was entirely normal.

Seven days later the ECG had returned to normal but during his convalescence salmonellae were again cultured from the stools and were present for a further 2 months. The organism was subsequently serotyped as Salmonella oranienburg, a bacterium recovered from the stools of 18 persons returning from holiday in Ibiza, all suffering from gastroenteritis (P.H.L.S. Communicable Disease Surveillance Centre-unpublished).

\section{Discussion}

In a review of 7,779 salmonella infections in man (excluding S. typhi), Saphra and Winter (1957) categorized four main clinical manifestations; gastroenteritis (68\%), a typhoid-like, septicaemic illness (8\%), focal manifestations (7\%) and the asymptomatic carrier state $(15 \%)$. The focal manifestations included 77 cases of meningitis $(0.8 \%)$ and 20 cases of endocarditis with pericarditis $(0 \cdot 2 \%)$. Salmonella meningitis is most commonly a disease of infants, particularly. the neonate, but the reason for the high susceptibility of this age group remains unclear. Birth trauma, immaturity of the gastrointestinal tract, decreased opsonization of Gram-negative bacteria by neonatal serum, and increased permeability of the blood-brain barrier are possible mechanisms (Cooke and Bell, 1922; Ziai and Haggerty, 1958). Henderson (1948) has reported that salmonella meningitis may occur as a superinfection, as five of his 144 cases followed episodes of meningococcal meningitis.

Twenty cases of salmonella meningitis in adults have been reported previously in the English literature (Kauffman and St Hilaire, 1979; Cherubin et al., 1981). All were sporadic but there appears to have been a change in the serotypes involved. Before 1940, eight of the nine cases described involved Salmonella typhi. However, of the 11 cases described since 1940 , only two were due to that organism. Despite antimicrobial therapy, salmonella meningitis in adults carries a mortality in excess of 50\% and it may reflect the fact that many such patients are immunocompromised. In 12 of the 20 cases reported, underlying conditions included alcoholism, Hodgkin's disease, renal failure and paraplegia.

Although chloramphenicol achieves high concentrations in the CSF and this patient's micro-organism was sensitive to it in vitro, previous experience indicates that chloramphenicol alone may be ineffective. Cherubin et al. (1981) commented that with chloramphenicol alone, three of the patients they reported relapsed or failed to respond completely and did so only after addition of ampicillin. Our patient appears to be unique in that he was previously healthy, yet developed not only septicaemia with meningitis but also carditis (manifested by pericarditis and a transitory abnormality of conduction). Although group $C$ serotype species of salmonella not uncommonly cause focal complications such as meningitis, the cardiac complications have not previously been described for this organism. Two minor outbreaks of $S$. oranienburg infection were reported last year: in Norway an outbreak of gastroenteritis followed consumption of black pepper contaminated with this organism. In addition, 18 patients developed enteritis associated with this organism while holidaying in Ibiza. The source of this infection is not known.

The reason for development of meningitis and carditis in our previously healthy, convalescent patient remains uncertain. We have no evidence of any underlying immune deficiency. However, it is known that neomycin can cause damage to the gastrointestinal mucosa. This is accompanied not only by changes in the mucosa which are visible under the light microscope, but also by breakdown of the mucosal barrier (Jacobson, Prior and Faloon, 1960; Cain, Reiner and Patterson, 1968). Since salmonella species are entero-invasive pathogens (Giannella, Formal and Dammn, 1973), it is possible that invasion of the blood stream leading to septicaemia and the other complications may have been aided by neomycininduced mucosal damage.

\section{Acknowledgment}

We wish to thank Dr A. M. Geddes for advice on the management of this patient.

\section{References}

Beene, M.L., Hansen, A.E. \& Fulton, M. (1951) Salmonella meningitis: Recovery from meningitis due to salmonella $\mathrm{sp}$. (type montevideo) with consideration of the problem of Salmonella meningitis. American Journal of Diseases of Children, 82, 567.

Cain, G.D., Reiner, E.B. \& Patterson, M. (1968) Effects of neomycin on disaccharidase activity of the small bowel. Archives of Internal Medicine, 122, 311.

Cherubin, C.E., MARr, J.S., Sierra, M.F. \& Becker, S. (1981) Listeria and gram negative bacillary meningitis in New York City, 1972-1979: frequent causes of meningitis in adults. American Journal of Medicine, 71, 199.

COOKE, J.V. \& BELL, H.H. (1922) The incidence of meningitis in early infancy, with a description of two cases due to unusual organisms. American Journal of Diseases of Children, 24, 387.

GHON, J. (1907) Bericht uber den XIV Internationalen Kongress fur Hygiene and Demographie (Berlin), 4, 21.

Giannella, R.A., Formal, S.B. \& Dammn, G.J. (1973) Pathogenesis of Salmonellosis; studies of fluid secretion, mucosal invasion, and morphologic reaction in the rabbit ileum. Journal of Clinical Investigation, 52, 441.

Henderson, L.L. (1948) Salmonella meningitis: Report of three cases and review of 144 cases from the literature. American Journal of Diseases of Children, 75, 351.

JACOBSON, E.D., PRIOR, J.T. \& FAloon, W.W. (1960) Malabsorption syndrome induced by neomycin; morphologic alterations in 
the jejunal mucosa. Journal of Laboratory and Clinical Medicine, 56, 345.

KAUfFMAN, C.A. \& ST HilaiRe, R.J. (1979) Salmonella meningitis: Occurrence in an adult. Archives of Neurology, 36, 578.

SAPHRA, I. \& WINTER, J.W. (1957) Clinical manifestations of salmonellosis in man: An evaluation of 7,779 human infectio identified at the New York Salmonella Center. New England Journal of Medicine, 256, 1128.

ZIAI, M. \& HAGGERTY, R.J. (1958) Neonatal meningitis. Nem England Journal of Medicine, 259, 314.

(Accepted 21 April 1983) 